\title{
Machines to support motor rehabilitation after stroke: 10 years of experience in Berlin
}

\author{
Stefan Hesse, MD; ${ }^{1 *}$ Henning Schmidt, Dipl-Ing; ${ }^{2}$ Cordula Werner, MA $^{1}$ \\ ${ }^{1}$ Klinik Berlin/Charité University Hospital, Department of Neurological Rehabilitation, Berlin, Germany; ${ }^{2}$ Fraunhofer \\ Institut-Institut für Produktionsanlagen und Konstruktionstechnik (IPK) Berlin, Germany
}

\begin{abstract}
The group at Klinik Berlin/Charite University Hospital in Berlin, Germany, began studies to promote motor recovery after stroke in the early 1990s. Following the introduction of treadmill training with partial body-weight support, the group designed an electromechanical gait trainer, GT I, based on movable foot plates that relieve therapist effort (e.g., when assisting the movement of the paretic limbs) and intensify patient gait training (GT). Preliminary results of a recent multicenter trial of 155 acute stroke patients showed that the GT I effectively promotes gait ability and competence in activities of daily living. The experimental group received $20 \mathrm{~min}$ of GT and 25 min of physiotherapy (PT) and the control group received 45 min of PT every day for 4 weeks. The laboratory's next step was the HapticWalker, a robotic walking simulator with freely programmable foot plates so that patients can, for example, additionally train for stair climbing and perturbations. The foot plates can be operated in full guidance or compliance control modes, thus reducing foot support according to the patient's learning success. For the severely affected upper limb, the laboratory's computerized arm trainer, called the Bi-Manu-Track, enables bilateral practice of forearm pronation/supination and wrist flexion/extension. Compared with electrical stimulation of the paretic wrist extensors, acute stroke patients with severe arm paresis $(n=44)$ had significantly more upper-limb muscle strength and control at the end of the 6-week intervention period and at follow-up. The laboratory's most recent and cost-effective development, the Nudelholz, is a purely mechanical device that bilaterally trains the shoulder, elbow, and wrist joints. It is intended for home therapy.
\end{abstract}

Key words: arm rehabilitation, controlled trials, gait, hemiparesis, locomotion, motor rehabilitation, physiotherapy, robotassisted therapy, robotics, stroke.

\section{INTRODUCTION}

We at the neurorehabilitation research laboratory at Klinik Berlin/Charité University Hospital began work in motor rehabilitation of stroke patients in the early 1990s. Guided by the task-specific treatment concept, we successfully introduced treadmill training with partial bodyweight support in the gait rehabilitation of chronic hemiparetic subjects [1]. In daily practice, the therapist effort required to assist patients' walking, e.g., when placing the paretic limbs, limits the intensity of therapy. Our next step was to develop the electromechanical gait trainer, GT I. On this machine, patients are secured with a harness and their feet placed on two driven foot plates whose movement simulates stance and swing. The robotic walking simulator, HapticWalker, continues this concept. It is based on the principle of programmable foot plates that allows the training of arbitrary foot motions, such as walking on level ground and climbing up and down stairs, based on real foot motion data. The control modes

Abbreviations: $3-\mathrm{D}=$ three-dimensional, $\mathrm{BATRAC}=$ Bilateral Arm Training with Rhythmic Auditory Cueing, DOF = degree of freedom, FAC = Functional Ambulatory Category, FES = functional electrical stimulation, FM $=$ Fugl-Meyer (Motor), GT = gait training, $\mathrm{PT}=$ physiotherapy.

*Address all correspondence to Stefan Hesse, MD; Klinik Berlin, Kladower Damm 223, 14089 Berlin, Germany; +4930-36503-105; fax: +49-30-36503-123.

Email: bhesse@zedat.fu-berlin.de

DOI: 10.1682/JRRD.2005.02.0052 
range from position-controlled to force- and compliancecontrolled motions during swing phase, and include simulation of perturbations.

For the upper limb, we developed the Bi-Manu-Track to treat severely affected stroke subjects who have no volitional distal wrist and finger activity. The Bi-ManuTrack enables the bilateral practice of two distal movement cycles. It is a robotic device offering different position and compliance control modes for therapy. Our latest, most cost-effective development, the purely mechanical Nudelholz ("rolling pin"), is intended for home therapy. This article presents the devices and related clinical studies for the motor rehabilitation of these upper and lower limbs.

\section{UPPER LIMB}

Following the pioneering work of Hogan et al. with the Massachusetts Institute of Technology (MIT)-Manus [2-3] and Burgar et al. with the Mirror Image Motion Enabler [4-5], we opted for a bilateral and more distal approach in both the Bi-Manu-Track and our latest design, the Nudelholz.

\section{Bi-Manu-Track}

The Bi-Manu-Track is a $2 \times 1$ degree-of-freedom (DOF) robot that enables hemiparetic patients to bilaterally practice two different movement cycles: forearm pronation/ supination and wrist flexion/extension (Figure 1) [6].

The robot handles perform a rocker-like rotary motion in an either a mirror-image or parallel fashion. Three different control modes are possible: passivepassive, active-passive (the nonaffected limb drives the affected limb), and active-active (the affected limb has to overcome an initial isometric resistance). Amplitude, speed, and resistances can be set individually. The users sit at a table with elbows bent $90^{\circ}$ and put their forearms in the midposition between pronation and supination into an arm trough. Each hand grasps a $3 \mathrm{~cm}$-diameter handle that is tapered at the top so it is easy to insert into the paretic hand. The hand is held in place by a $6 \mathrm{~cm}$ Velcro strap. The handles are connected by an axis linked to the respective position-/torque-controlled motor. Two handle sets are available: one with a horizontal axis of rotation for the elbow motion and one with a vertical axis for the wrist motion. To switch motion direction (i.e., from

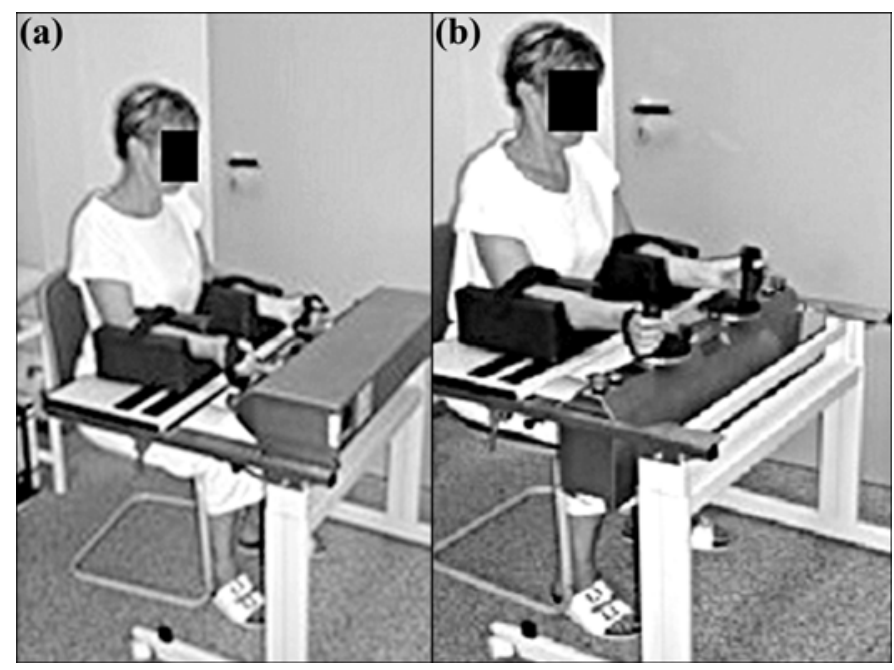

Figure 1.

Bi-Manu-Track computerized arm trainer for practicing (a) bilateral forearm pronation/supination and (b) wrist flexion extension.

elbow to wrist), the user tilts the device $90^{\circ}$ downward and exchanges the handles.

Each of the two drives provides torque up to $5 \mathrm{~N} \bullet \mathrm{m}$. Position control and retractive drive forces regulate the online registration of position and strength; a computer collects the data and controls the drives. A display shows the number of training cycles performed. The user selects operation mode, side of hemiparesis, range, speed, and the resistance of movements on a digital control unit $800 \times 320 \times 220 \mathrm{~mm}$ positioned between the two handles. The device can be fixed on any table but is ideally placed on a height- and slope-adjustable therapy table for optimal positioning of the user. A $230 \mathrm{~V}$ power supply is required. Implemented safety features comply with European standards for medical devices-mainly electromagnetic testing, leaking currents of less than $1 \mathrm{~mA}$, a mechanical breaking of the movement when the torque exceeds $4 \mathrm{~N} \bullet \mathrm{m}$, emergency brakes within reach of the users, skin-friendly materials, and minimal contusion risk.

The assumptions we made about the underlying mechanisms that influenced our design were that (1) the bilateral approach would facilitate the paretic side via intercallosal fibers [7], (2) the more distal movements have a larger cortical representation of the hand and fingers, and (3) proximal upper-limb segments, if trained, would compete with distal upper-limb segments for brain plasticity [8]. This competition means that the treatment should begin distally, because a preferential proximal 
treatment approach might even impede the restoration of the paretic hand and fingers.

Open clinical studies in severely affected chronic patients documented a reduction in muscle tone for the wrist and finger joints and a minor improvement in motor functions without clinical relevance following 4 weeks of daily training [6]. The patients unanimously recommended the therapy, appreciated that the therapy focused more on the paretic hand than the shoulder girdle, and noticed decreased muscle tone. We restricted the number of repetitions (800 per session evenly distributed in the four movement directions) to prevent wrist pain and swollen hands. Shoulder pain was not a problem.

A subsequent randomized trial of two centers included 44 acute stroke patients whose first strokes were 4 to 8 weeks before the study [9]. All were severely affected, with initial Fugl-Meyer (FM) Motor score (0-66) of $<18$ and no volitional activity of the wrist and finger extensors. For 6 weeks, the randomly assigned patients practiced either with the robot or electrical stimulation of the paretic wrist extensors. The FM Motor score was the primary outcome parameter and was blindly assessed with the help of a standardized video. Both groups initially had homogenous clinical data (the muscle strength and tone were secondary). Over time, both groups significantly improved their upper-limb motor control and power; the between-group comparison revealed superior results in the robot-trained group both at the end of the study and at 3-month follow-up later. The muscle tone did not change and side effects did not occur. Interestingly, the proximal (0-42) and distal (0-24) FM Motor subscores improved evenly in the robot-trained group, i.e., the treatment effect was generalized (Figure 2).

The robot-trained group practiced a total of 24,000 repetitions (evenly distributed in the four movement directions) as compared with 1,800 to 2,400 repetitions of one movement (wrist extension) in the electrical stimulation group. In addition, the robot therapy was bilateral and aimed at facilitating the paretic side via intercallosal fibers.

\section{Nudelholz}

Our recent work concentrated on the development of inexpensive home exercise machines for severely affected stroke patients. We developed the purely passive mechanical device, Nudelholz (Figure 3), with 3 DOF (2 translatory and 1 rotatory), again following the approach of bilateral and distal training. With this device, stroke patients can train three bilateral movement cycles: elbow
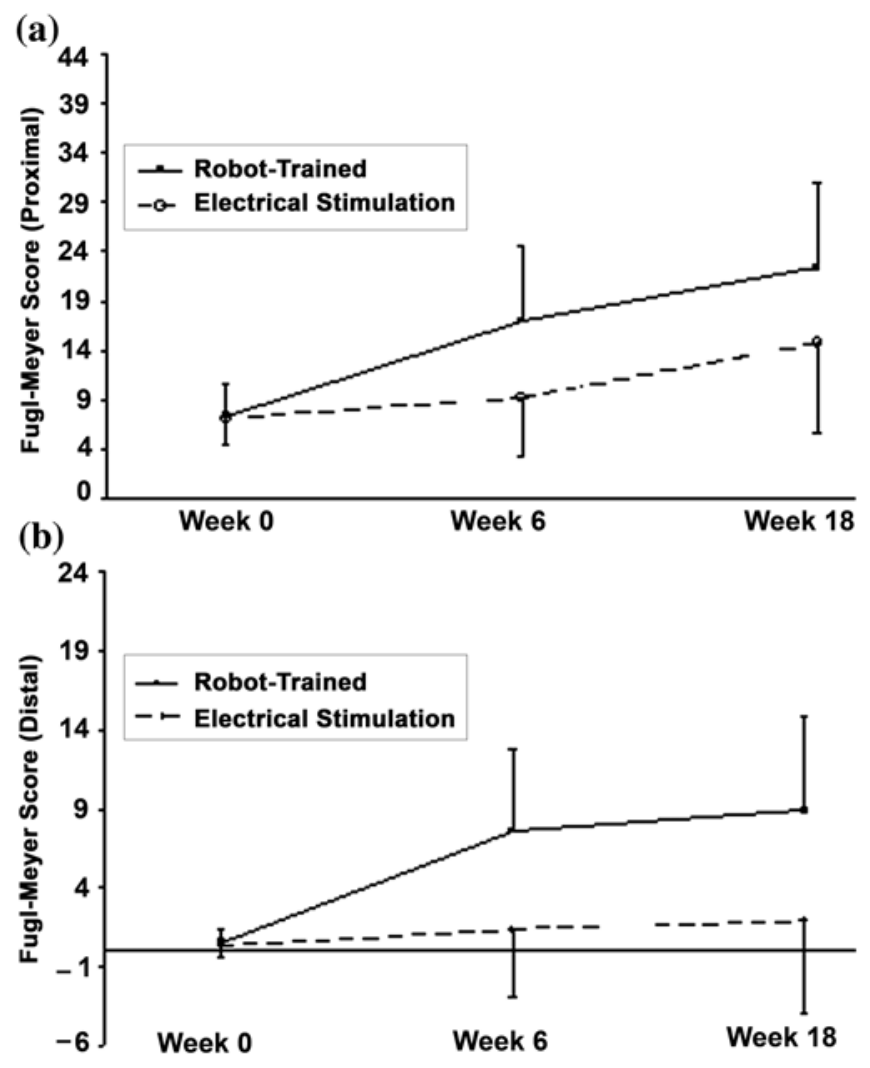

Figure 2.

Mean \pm standard deviation of (a) proximal (0-42) and (b) distal (0-24) subscores of Fugl-Meyer upper-limb motor score (0-66) for Bi-ManuTrack group (robot-trained) and electrical stimulation group.

flexion/extension, shoulder abduction/adduction, and wrist flexion/extension, either isolated or combined. The patient grasps two handles (either actively or with the help of Velcro) so the movement of the nonaffected side guides the affected hand and arm. An optional cordless computer mouse can be attached to the Nudelholz middle bar. Then the 2 DOF translatory motion of the user-driven bar (forward/backward and left/right) is transmitted to the attached mouse. The mouse movement can be visualized on a computer screen for incorporating visual biofeedback into therapy. Initial clinical case studies revealed significantly decreased muscle tone and improved upper-limb motor control, even in chronic patients. These results confirm previous reports about the application of a similar custom-made device, called the Bilateral Arm Training with Rhythmic Auditory Cueing (BATRAC) [10]. The BATRAC only has 1 DOF per hand, allowing a bilateral movement of two handles forward and backward. 


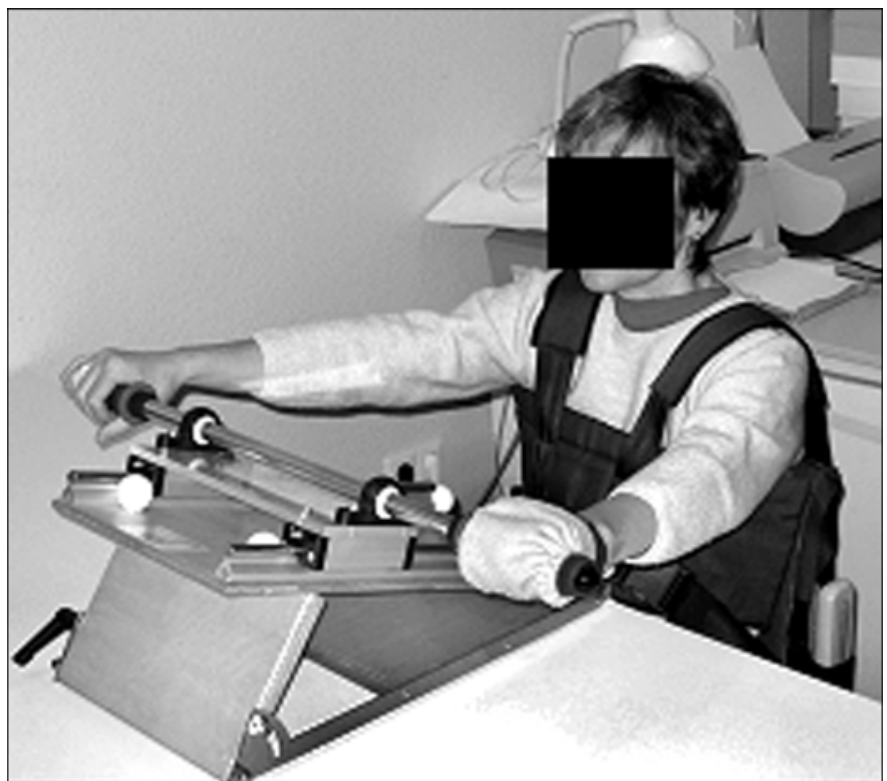

Figure 3.

Nudelholz mechanical arm trainer for practicing bilateral shoulder, elbow, and wrist movement. Nonaffected limb drives affected limb.

\section{LOWER LIMB}

Gait machines are designed for relieving physiotherapist strain (e.g., when assisting the paretic limbs or manually controlling the weight shift on the treadmill or floor) and intensifying patient gait training (GT). So far, two different designs have been used clinically: (1) a treadmill with a driven gait orthosis [11-12] and (2) a moving foot plate system.

\section{GT I Gait Trainer}

The electromechanical gait trainer, GT I (Figure 4), is based on a moving foot plate system [13]. On this machine, the harness-secured patient is positioned on two foot plates, the movements of which simulate stance and swing at a fixed ratio of 60 to 40 percent with the help of a sun-gear system. The cadence and step length $(28-48 \mathrm{~cm})$ can be set individually. Ropes attached to the harness from above and at the pelvis are connected to the drive system via pulleys and gears that control the sinusoidal movement of the center of mass in vertical and lateral directions. Multichannel cycle-dependent functional electrical stimulation (FES) is optional; i.e., the machine can trigger an external, commercially available, programmable 8-channel stimulator (Motionstim, Krauth and Timmer-

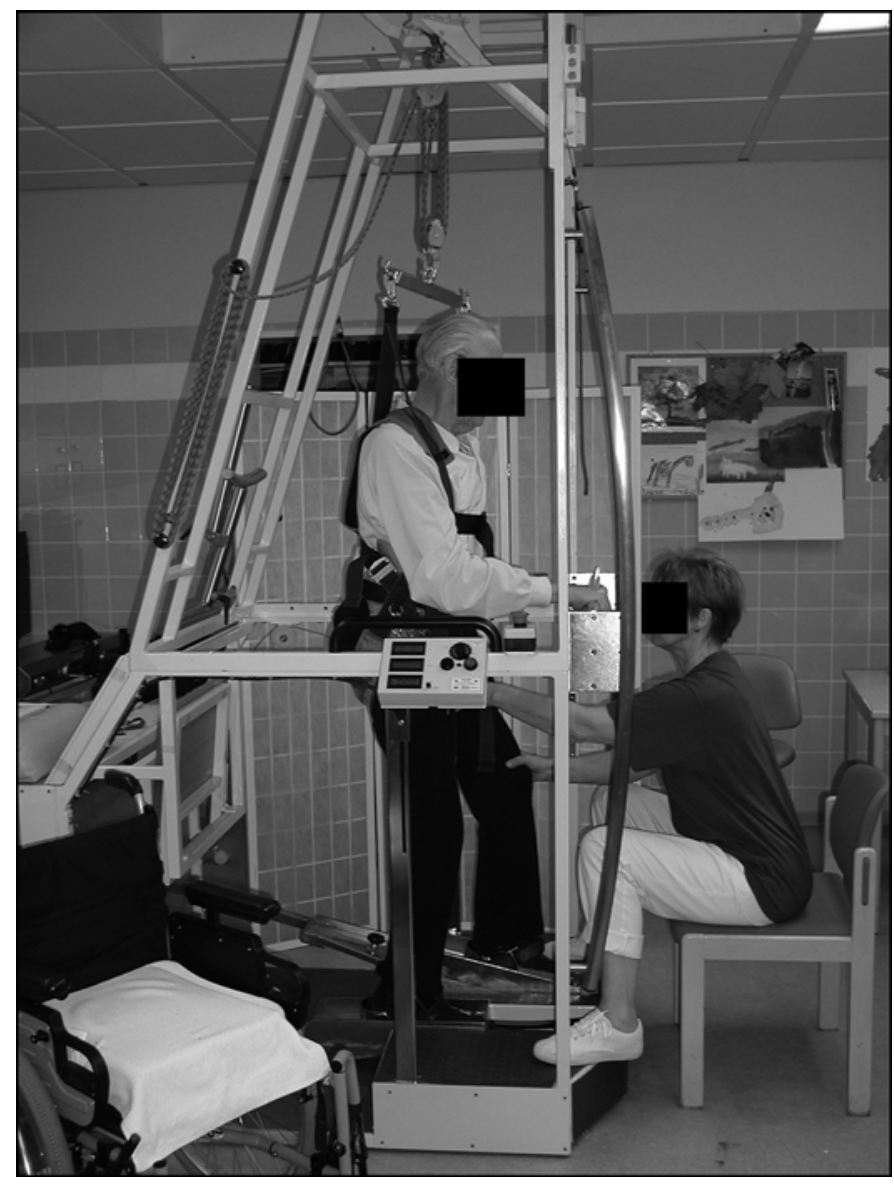

Figure 4.

GT I electromechanical gait trainer. Harness-secured patient is positioned on two foot plates that when moved simulate stance and swing.

man, Germany) during the stance and/or swing phase, depending on programming. Therapists use FES, for instance, to stabilize the knee during stance phase (quadriceps, biceps femoris), the pelvis (gluteus medius), or to support push-off of the ankle (gastrocnemius).

The first stroke studies revealed improved gait ability and lower-limb muscle activity in chronic, nonambulatory stroke patients following 4 weeks of applied GT I training [14]. A randomized crossover study included 30 acute patients who either followed an $\mathrm{ABA}$ or $\mathrm{BAB}$ design in which phase A was 2 weeks of GT I and phase B was 2 weeks of manually assisted treadmill training. In the last treatment phase, ABA patients reached a significantly higher gait level, while walking velocity and other motor functions did not differ. Only one therapist-instead of two or even three-assisted these patients' gait on the 
machine, sitting in front of the patients and guiding their paretic knees [15].

A recent multicenter trial, the DEutsche GAngtrainerStudie (DEGAS), included 155 acute nonambulatory stroke patients [16]. Following random group allocation, patients in the experimental group (Group A) practiced 20 min of GT on the GT I and 25 min of physiotherapy (PT) and patients in the control group (Group B) practiced 45 min of PT 5 days a week for 4 weeks. The PT in both groups concentrated on repetitive GT on the floor and stairs. Gait ability, assessed by the Functional Ambulation Category (FAC) (0-5 scale on which 0 describes a patient who cannot walk or requires help of two or more therapists and 5 describes a patient who is ambulatory under all conditions), and competence in basic activities of living (Barthel Index, 0-100) were the primary blindly assessed variables. Both groups had homogeneous clinical data at study onset. Group A patients scored significantly higher at the end of the study and at follow-up on both primary outcome parameters [16] (Figure 5). At the end of the study, 41 of 77 (53.2\%) in Group A versus 17 of 77 (22.1\%) in Group B could walk independently, i.e., they had reached an FAC level of either 4 or 5 .

Group A patients practiced 800 to 1,200 steps each session on the machine, while Group B patients rarely exceeded 200 steps during their individual 45 min PT sessions. Accordingly, the known positive correlation between treatment intensity and motor outcome most likely explained the superior treatment result [17]. Relevant side effects did not occur.

Patients with spinal cord injury could benefit from GT I training as well. Chronic patients with American Spinal Injury Association (ASIA) classifications C and D markedly improved their gait ability and walking velocity during a 5-week daily training program and more than doubled gait speed and endurance. The dynamic electromyogram revealed increased activity of the gastrocnemius muscle and a more physiological lower-limb muscle activation pattern of the thigh muscles. Therapists rated the program less strenuous compared with manually assisted treadmill training [18].

\section{HapticWalker}

The HapticWalker (Figure 6) is our group's latest development for gait rehabilitation [19]. It was designed and built in collaboration with the Fraunhofer InstitutInstitut für Produktionsanlagen und Konstruktionstechnik in Berlin, Germany. The device is a robotic walking simu-

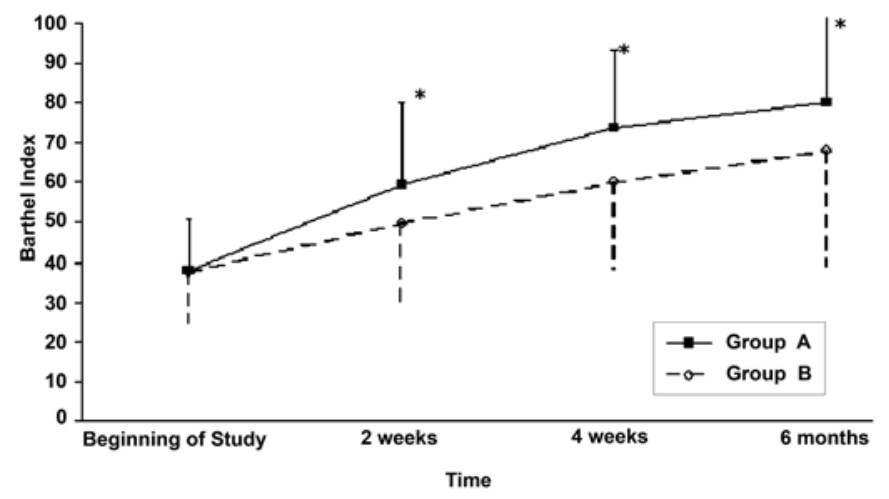

Figure 5.

DEutsche GAngtrainer-Studie (DEGAS): mean \pm standard deviation Barthel Index (0-100) of Group A (experimental group) and Group B (control group) over time. ${ }^{*}$ Significant difference between groups, $p<$ 0.05 .

lator for gait rehabilitation based on the principle of programmable foot plates; i.e., it continues the successfully applied approach of movable foot plates and allows patient-physiotherapist interaction during training. The patient's feet are fixed onto the foot plate end effectors of two freely programmable robot arms on the HapticWalker, while his or her trunk is secured by a patientlift-driven harness system. Our goal for the HapticWalker project was to design and build a highly dynamic robotic walking simulator that would allow the realistic simulation of arbitrary natural gait patterns regarding position and velocity profiles to achieve optimal afferent input to the central nervous system. The machine has the following main advantages compared with the GT I: (1) The foot plate trajectories are freely programmable, hence patient training is no longer restricted to walking on level ground. Arbitrary foot trajectories-for instance, walking up or down stairs - can also be trained. (2) The foot trajectory motion profiles are based on measured free-walking foot-motion data at normal walking speeds, which can be fully adjusted to individual patients' needs. (3) Six DOF force/torque sensors are located under each foot plate, the readings from which are the basis for different types of force/compliance control. In addition, they are used for diagnosis during the stance and swing phases (during the stance phase, the 6 DOF sensor readings comprise the ground reaction forces, i.e., a comprehensive gait analysis can be done automatically during patient training on the machine). (4) Motion generation algorithms for different types of artificial foot motions are implemented, for 


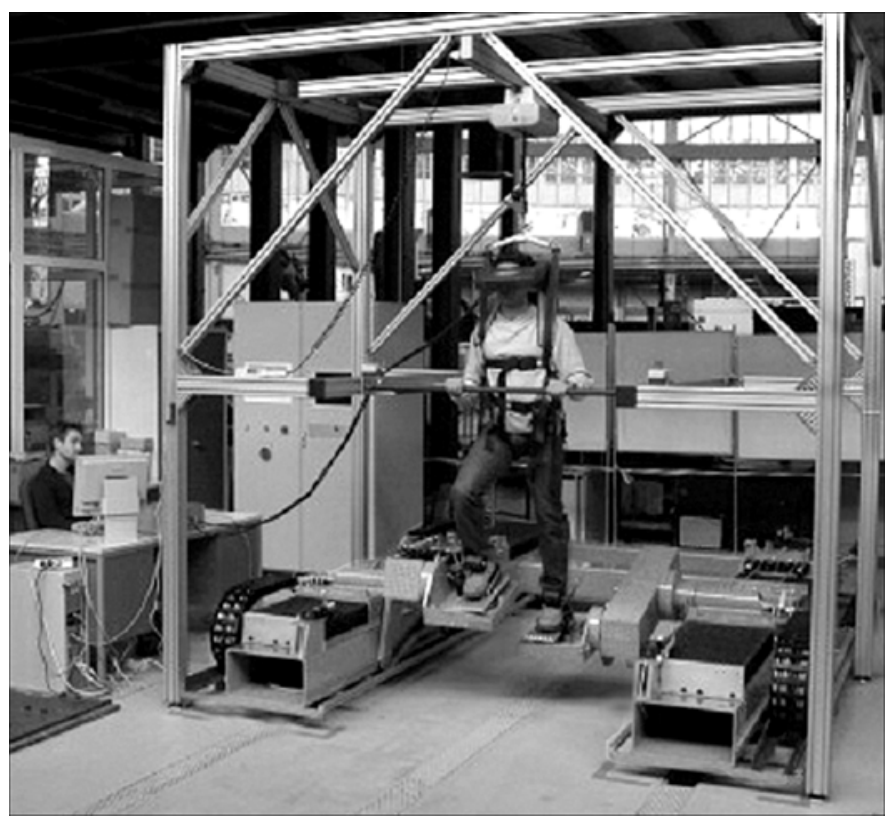

Figure 6.

HapticWalker, robotic walking simulator for gait training to practice individual and arbitrary walking trajectories (e.g., walking on level ground, climbing up/down stairs, perturbations).

example, perturbations such as stumbling or sliding during GT. The foot plate motion can also be switched to fully artificial motion for training or diagnosis (e.g., simulating cycling or performing even different trajectories on right and left foot plate). (5) This generic haptic foot device can be integrated into virtual GT environments and combined with other modalities (e.g., visual feedback) for enhanced research and training.

The first step during HapticWalker development was the analysis of different daily-life foot trajectories, such as walking on level ground, upstairs, or downstairs at walking speeds up to $5 \mathrm{~km} / \mathrm{h}$ and cadences up to $120 \mathrm{steps} /$ min, hence covering the normal range of adult walking speeds, step lengths, and step heights. This analysis revealed that trunk-relative foot movement is cyclic and has a highly variable path velocity [20], with maximum path velocities of $3 \mathrm{~m} / \mathrm{s}$ during mid swing phase and maximum accelerations/decelerations up to $35 \mathrm{~m} / \mathrm{s}^{2}(3.5 \mathrm{~g})$ at heel contact as well as push-off during the stance phase. The most suitable mathematical description of this Cartesian cyclic motion was found to be a higher order Fourier series (at minimum 6th order for cadences up to 120 steps/ s). Furthermore, the machine should be able to realistically simulate perturbations, such as stumbling or sliding.
A major advantage of the HapticWalker is that it allows realistic training of the foot trajectories needed in daily life, which cannot be trained during conventional gait rehabilitation that is usually restricted to relatively smooth walking on level ground (the clinic floor or treadmill). In addition to the required foot plate workspace and dynamics covering all desired foot trajectories, a number of important design issues concerned patient transfer onto and off the machine, as well as patient and operator safety. Safety was a major issue in general, since the HapticWalker was designed to be an interactive machine that did not impede the therapist's access to the patient. We did not intend the HapticWalker to fully substitute for the physiotherapist but only to relieve him or her from strenuous work and improve GT for the patient. Furthermore, we decided to build the device as a modular robot kinematics, starting with 3 DOF per foot plate in the sagittal plane, with an optional extension to 6 DOF for full three-dimensional (3-D) foot motion, plus an additional drive to support the metatarsal joint motion (relative motion between fore- and hind foot). These demands required a custommade robotic walking simulator. The robot arms are based on hybrid parallel-serial kinematics, which combine the main advantages of parallel (i.e., high dynamics) and serial (i.e., good workspace-to-machine-size ratio) mechanisms. In addition, high dynamics and backdriveability were optimized by the application of electrical direct-drive motors.

A full-featured robot-control software based on RTLinux has been developed as well. This software is based on the proven software architecture of industrial robot controllers, including features like multithreaded real-time control, user instruction set, user programs, command line interpreter, and real-time communication to operator PC. In addition, we developed a number of algorithms for the synthesis and real-time interpolation of arbitrary cyclic walking trajectories. Thus, we are able to perform individually adjusted foot trajectories on the HapticWalker in any respect (step length, step height, angular profile, cadence, velocity). The algorithm applies the mathematical description of Fourier series to cyclic foot motions. Those are best suited to this type of motion compared wih other mathematical descriptions (e.g., polynomial, spline). Furthermore, special algorithms based on the automatic online adaptation of the motion speed override were developed for the realistic simulation of perturbations such as stumbling and sliding. These asynchronous events can be triggered by the physiotherapist at any time during GT. 
The physiotherapist chooses appropriate foot trajectory parameters via an intuitive graphical user interface software on a dedicated Windows operator PC. The software was especially designed for use by nontechnical personnel and allows safe machine operation in any respect. The operator is given a number of graphical elements (buttons, sliders, edit controls) for gait trajectory adjustment to individual patient's needs based on captured real-world foot trajectories. First, the operator chooses a basic foot trajectory (plane ground, upstairs, downstairs) and then adjusts the necessary gait parameters (step length, step height, angular profile, cadence). Then the user software automatically calculates a full natural foot trajectory and sends its Fourier coefficients to the robot controller. In addition, the user software displays various online measurement values (positions, forces, etc.) in two-dimensional plots and an intuitive integrated 3-D real-time animation view.

The data from 6 DOF force/torque sensors under each foot plate are used by the robot controller for an admittance-control-based force/compliance-control scheme as well as patient-driven adaptive walking speed control. During the position-controlled mode, the machine fully guides the patient, which is necessary during the initial rehabilitation phase. When applying compliant foot plate behavior, the machine gradually decreases its support, depending on the patient's learning success, which leads to so-called "patient-cooperative training” [21]. When applying zero-force control during the swing phase, the machine acts like a classical haptic device that is completely passive above the ground and fully driven by the user. In this mode, only virtual ground conditions are simulated. Force control also enables the application of artificial force fields during any phase of gait [22-23]. Furthermore, the force sensor readings allow continuous monitoring and documeting the patient's learning success during all phases of gait.

The first HapticWalker prototype was successfully completed and tested with nondisabled subjects and was recently approved by the German technical inspectorate for medical devices. Clinical trials with hemiparetic subjects will begin soon.

An important area of further development will be the optimization of force/compliance control algorithms based on the clinical trial results. Another working area will be the extension of gait motion generation algorithms to the training of arbitrarily combined virtual training paths. This could be complemented by combining the haptic foot sensations with visual virtual reality for showing the patient a 3-D view of the virtual training path.

\section{CONCLUSIONS}

Machines open new dimensions in motor rehabilitation after stroke, with smaller drives and more sophisticated man-machine interactions to come. To promote this new field, more randomized clinical studies are mandatory. Technology is fascinating, but every piece requires clinical evaluation to avoid the risk of an "art for art's sake" style of research. On the other hand, therapists' fears of becoming jobless must be addressed. Initially, purely technical rehabilitation without the human touch was imagined. However, the therapists we have worked with over the years have come to realize the potential benefits of machines to their patients and themselves. In the studies presented here, machines were always supplementary tools that assisted the therapist and enabled more intensive practice, thereby improving treatment. These studies were also in line with modern concepts of motor rehabilitation of stroke patients. While no machine can ever substitute for the "human touch" of an experienced therapist, we look forward to the future development of this exciting new field of rehabilitation.

\section{ACKNOWLEDGMENTS}

This material was based on work supported in part by The Bundesmisterium für Bildung und Forschung, grant BEO 11.

Reha-Stim, Berlin, Germany, holds the national patents of the GT I gait trainer, Bi-Manu-Track, and the Nudelholz. The company is owned by Dr. Beate BrandlHesse, the spouse of the first author.

\section{REFERENCES}

1. Hesse S, Bertelt C, Jahnke MT, Schaffrin A, Baake P, Malezic M, Mauritz KH. Treadmill training with partial body weight support compared with physiotherapy in nonambulatory hemiparetic patients. Stroke. 1995;26(6): 976-81. [PMID: 7762049]

2. Hogan N, Krebs HI, Charnarong J, Sharon A, inventors. Massachusetts Institute of Technology, assignee. Interactive 
robotics therapist. United States patent US 5466213. 1995 Nov 14.

3. Volpe BT, Krebs HI, Hogan N, Edelstein L, Diels C, Aisen ML. A novel approach to stroke rehabilitation: Robot-aided sensorimotor stimulation. Neurology. 2000;54(10):1938-44. [PMID: 10822433]

4. Burgar CG, Lum PS, Shor P, Van der Loos M. Development of robots for rehabilitation therapy: The Palo Alto VA/Stanford experience. J Rehabil Res Dev. 2000;37(6): 663-73. [PMID: 11321002]

5. Lum PS, Burgar CG, Shor PC, Majmundar M, Van der Loos M. Robot-assisted movement training compared with conventional therapy techniques for the rehabilitation of upper-limb motor function after stroke. Arch Phys Med Rehabil. 2002;83(7):952-59. [PMID: 12098155]

6. Hesse S, Schulte-Tigges G, Konrad M, Bardeleben A, Werner C. Robot-assisted arm trainer for the passive and active practice of bilateral forearm and wrist movements in hemiparetic subjects. Arch Phys Med Rehabil. 2003;84(6): 915-20. [PMID: 12808550]

7. Förster O, Buhnke O. Handbuch der neurologie, band 6 . Berlin (Germany): Springer-Verlag; 1936.

8. Muellbacher W, Richards C, Ziemann U, Wittenberg G, Weltz D, Boroojerdi B, Cohen L, Hallett M. Improving hand function in chronic stroke. Arch Neurol. 2002;59(8): 1278-82. [PMID: 12164724]

9. Hesse S, Werner C, Pohl M, Rueckriem S, Mehrholz J, Lingnau ML. Computerized arm training improves the motor control of the severely affected arm after stroke: A single-blinded randomized trial in two centers. Stroke. 2005; 36(9):1960-66. [PMID: 16109908$]$

10. Whitall J, McCombe Waller S, Silver KH, Macko RF. Repetitive bilateral arm training with rhythmic auditory cueing improves motor function in chronic hemiparetic stroke. Stroke. 2000;31(10):2390-96. [PMID: 11022069$]$

11. Colombo G, Joerg M, Schreier R, Dietz V. Treadmill training of paraplegic patients using a robotic orthosis. J Rehabil Res Dev. 2000;37(6):693-700. [PMID: 1132005]

12. HealthSouth Corporation. Powered gait orthosis and method of utilizing same. United States patent US 6689075. 2004 Feb.

13. Hesse S, Sarkodie-Gyan T, Uhlenbrock D. Development of an advanced mechanised gait trainer, controlling movement of the centre of mass, for restoring gait in non-ambulant subjects. Biomed Tech (Berl). 1999;44(7-8):194-201. [PMID: 10472726]

14. Hesse S, Werner C, Uhlenbrock D, Von Frankenberg S, Bardeleben A, Brandl-Hesse B. An electromechanical gait trainer for restoration of gait in hemiparetic stroke patients: Preliminary results. Neurorehabil Neural Repair. 2001; 15(1):39-50. [PMID: 11527278]

15. Werner C, Von Frankenberg S, Treig T, Konrad M, Hesse S. Treadmill training with partial body weight support and an electromechanical gait trainer for restoration of gait in subacute stroke patients: A randomized crossover study. Stroke. 2002;33(12):2895-2901. [PMID: 12468788]

16. Pohl M, Werner C, Holzgraefe M, Kroczek G, Mehrholz J, Wingendorf I, Hoellig G, Koch R, Hesse S. Repetitive locomotor training and physiotherapy improve walking and basic activities of daily living after stroke: A single-blind, randomised, multi-centre trial (Deutsche Gangtrainerstudie, DEGAS). Clin Rehabil, 2006, In press.

17. Kwakkel G, Wagenaar RC, Twisk JW, Lankhorst GJ, Koetsier JC. Intensity of leg and arm training after primary middle-cerebral-artery stroke: A randomised trial. Lancet. 1999;354(9174):191-96. [PMID: 10421300]

18. Hesse S, Werner C, Bardeleben A. Electromechanical gait training with functional electrical stimulation: Case studies in spinal cord injury. Spinal Cord. 2004;42(6):346-52. [PMID: 14993895]

19. Schmidt H, Sorowka D, Hesse S, Bernhardt R. Development of a robotic walking simulator for gait rehabilitation. Biomed Tech (Berl). 2003;48(10):281-86. [PMID: 14606269]

20. Schmidt H, Hesse S, Bernhardt R, Krüger J. HapticWalkerA novel haptic foot device. ACM Trans Appl Percept. 2005;2(2):166-80.

21. Riener R, Lunenberger L, Colombo G. Cooperative strategies for robot-aided gait neuro-rehabilitation. In: Proceedings of the 26th Annual International Conference of IEEE Engineering in Medicine and Society, Vol 7; 2004 Sep 1-5; San Francisco, CA. New York: IEEE; 2004. p. 4822-24.

22. Scheidt RA, Reinkensmeyer DJ, Conditt MA, Rymer WZ, Mussa-Ivaldi FA. Persistence of motor adaptation during constrained, multi-joint, arm movements. J Neurophysiol. 2000;84(2):853-62. [PMID: 10938312]

23. Emken JL, Reinkensmeyer DJ. Accelerating motor adaptation by influencing neural computations. In: Proceedings of the 26th Annual International Conference of IEEE Engineering in Medicine and Biology Society, Vol 6; 2004 Sep 1-5; San Francisco, CA. New York: IEEE; 2004. p. 4033-36.

Submitted for publication February 25, 2005. Accepted in revised form March 6, 2006. 